\title{
Mit welchem BMI lebt man am längsten?
}

Es ist bekannt, dass ein hoher Body-Mass-Index (BMI) mit erhöhter Mortalität für kardiovaskuläre Erkrankungen und verschiedene Krebserkrankungen einhergeht. Hier sind vor allem das Körperfett und besonders das viszerale Fett von entscheidender Bedeutung. Unklar ist allerdings die direkte Verbindung zwischen BMI und Gesamtmortalität.

- Verschiedene Berichte zeigten in der Vergangenheit, dass ein niedriger wie auch ein hoher BMI die Morbidität und Mortalität erhöht und dass es unterschiedliche Einflüsse auf diesen Prozess bei Rauchern gibt.

In einer neuen Studie wurden jetzt die gepoolten Daten von 19 prospektiven Studien, die insgesamt nahezu 1,5 Millionen weiße Erwachsene im Alter von 19 bis 84 Jahren erfassten, ausgewertet. Dabei wurde die Assoziation BMI und Gesamtmortalität, adjustiert für Alter, körperliche Aktivität, Alkoholkonsum, Bildungsstatus und Lebenssituation, untersucht. Mithilfe von Kurz-Repressionsanalysen wurde das BMI-bezogene Mortalitätsrisiko bestimmt.
Der mediane Ausgangs-BMI war 26,2. Während eines mittleren Verlaufs von zehn Jahren konnten 160087 Todesfälle identifiziert werden.

Interessanterweise konnte eine Assoziation von niedrigem BMI (kleiner 20) und erhöhter Mortalität beobachtet werden, wozu bereits bestehende Erkrankungen verantwortlich gemacht wurden, da nach dem Follow-up dieser Zusammenhang deutlich schwächer war. Eine Subanalyse untersuchte Raucher im Vergleich zu Nichtrauchern. Hier zeigte sich ein höheres BMI-abhängiges Mortalitätsrisiko für Nichtraucher.

- A.Berrington de Gonzalez et al. Body-mass index and mortality among 1.46 million white adults. N. Engl. J. Med. 363 (2010): 2211-2219

\begin{tabular}{|l|l|}
\hline Tabelle 1 & $\begin{array}{l}\text { Mortalität bei Frauen } \\
\text { (Cl= confidence interval) }\end{array}$ \\
\hline $15.0-18.4$ & $1.47(95 \%-\mathrm{Cl}, 1.33-1.62)$ \\
\hline $18.5-19.9$ & $1.14(95 \%-\mathrm{Cl}, 1.07-1.22)$ \\
\hline $20.0-22.4$ & $1.00(95 \%-\mathrm{Cl}, 0.96-1.04)$ \\
\hline $25.0-29.9$ & $1.13(95 \%-\mathrm{Cl}, 1.09-1.17)$ \\
\hline $30.0-34.9$ & $1.44(95 \%-\mathrm{Cl}, 1.38-1.50)$ \\
\hline $35.0-39.9$ & $1.88(95 \%-\mathrm{Cl}, 1.77-2.00)$ \\
\hline $40.0-49.9$ & $2.51(95 \%-\mathrm{Cl}, 2.30-2.73)$ \\
\hline
\end{tabular}

\section{Kommentar}

Es besteht ein klarer Zusammenhang zwischen BMI und Gesamtmortalität. Interessanterweise trat dieser Zusammenhang bei Rauchern in hohen BMI-Bereichen schwächer, bei niedrigem BMI unter 25 hingegen deutlich stärker zu Tage. Insgesamt bestand ein direkter Zusammenhang zwischen BMI und Gesamtmortalität mit dem besten BMI-Bereich zwischen 20 und 22,4 $\mathrm{kg} / \mathrm{m}^{2}$. Allerdings wurden ausschließlich Kaukasier untersucht. Die Daten sind nicht direkt extrapolierbar auf andere ethnische Gruppen. Die Quintessenz dieser Studie ist: BMI unter 25 rettet Leben! P. SCHWARZ = 